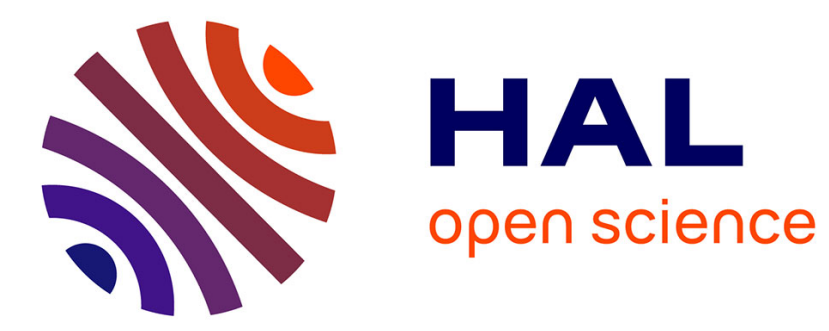

\title{
Low-resolution Electromagnetic Brain Tomography (LORETA) of Monozygotic Twins Discordant for Chronic Fatigue Syndrome
}

Leslie Sherlin, Thomas Budzynski, Helen Kogan Budzynski, Marco Congedo, Mary E. Fisher, Dedra Buchwald

\section{To cite this version:}

Leslie Sherlin, Thomas Budzynski, Helen Kogan Budzynski, Marco Congedo, Mary E. Fisher, et al.. Low-resolution Electromagnetic Brain Tomography (LORETA) of Monozygotic Twins Discordant for Chronic Fatigue Syndrome. NeuroImage, 2007, 34 (4), pp.1438-42. hal-00460501

\section{HAL Id: hal-00460501 https://hal.science/hal-00460501}

Submitted on 1 Mar 2010

HAL is a multi-disciplinary open access archive for the deposit and dissemination of scientific research documents, whether they are published or not. The documents may come from teaching and research institutions in France or abroad, or from public or private research centers.
L'archive ouverte pluridisciplinaire HAL, est destinée au dépôt et à la diffusion de documents scientifiques de niveau recherche, publiés ou non, émanant des établissements d'enseignement et de recherche français ou étrangers, des laboratoires publics ou privés. 
Running head: LORETA in CFS Twins

\title{
Low-resolution Electromagnetic Brain Tomography (LORETA) of Monozygotic Twins Discordant for Chronic Fatigue Syndrome
}

\author{
Leslie Sherlin, MS \\ Thomas Budzynski, Ph.D. \\ Helen Kogan Budzynski, Ph.D. \\ Marco Congedo, Ph.D. \\ Mary E. Fischer, Ph.D. \\ Dedra Buchwald, M.D.
}

From the Harold Able School of Psychology, Capella University, Minneapolis, MN, and Nova Tech EEG, Inc.(Mr. Sherlin), the Departments of Psychosocial and Community Health (Drs. Budzynski and Kogan Budzynski) and Medicine (Dr. Buchwald), University of Washington, Seattle, WA, France Telecom Research \& Development, Grenoble, France (Dr. Congedo), and the Division of Epidemiology and Biostatistics, University of Illinois, Chicago (Dr. Fischer).

Correspondence and reprint requests should be addressed to: Leslie Sherlin, 8503 E Keats Ave, Mesa, AZ 85208, LeslieSherlin@NovaTechEEG.com.

This work was partially funded by grant U19AI38429 from the National Institutes of Health (Dr. Buchwald) and Nova Tech EEG, Inc. 


\section{ABSTRACT}

Background: Previous work using quantified EEG has suggested that brain activity in individuals with chronic fatigue syndrome (CFS) and normal persons differs. Our objective was to investigate if specific frequency band-pass regions and spatial locations are associated with CFS using low-resolution electromagnetic brain tomography (LORETA).

Methods: We conducted a co-twin control study of 17 pairs of monozygotic twins where 1 twin met criteria for CFS and the co-twin was healthy. Twins underwent an extensive battery of tests including a structured psychiatric interview and a quantified EEG. Eyes closed EEG frequencydomain analysis was computed and the entire brain volume was compared of the CFS and healthy twins using a multiple comparison procedure.

Results: Compared with their healthy co-twins, twins with CFS differed in current source density. The CFS twins had higher delta in the left uncus and parahippocampal gyrus and higher theta in the cingulate gyrus and right superior frontal gyrus.

Conclusions: These findings suggest that neurophysiological activity in specific areas of the brain may differentiate individuals with CFS from those in good health. These preliminary findings await replication.

Keywords: Chronic fatigue syndrome, twins, electroencephalography, low resolution electromagnetic tomography, LORETA 


\section{Introduction}

Chronic fatigue syndrome (CFS) is an illness characterized by profound fatigue lasting at least 6 months accompanied by disturbances of sleep, mood, musculoskeletal pain, and other symptoms (Fukuda et al., 1994). CFS patients are often depressed (Manu , Matthews, \& Lane,, 1989; Wood, Bentall, Gopfert, \& Edwards, 1991; Katon, Buchwald, Simon, Russo, \& Mease, 1991) and report problems with forgetfulness, attention, and information processing (Komaroff and Buchwald, 1991; 1998). Investigators have sought, mostly unsuccessfully, to characterize these complaints by linking them to specific changes on objective measures of brain function. Recently, EEG methods have been used to examine central nervous system function in which brain wave electrical patterns from multiple scalp electrodes are quantified by computer programs. In one study using quantified EEG, CFS patients had higher theta power than healthy control subjects (Billiot, Budzynski, \& Andrasik, 1997). In another, spectral analysis suggested that physical activity-induced EEG signal changes might serve as physiological markers for CFS (Siemionow, Fang, Calabrese, Sahgal, \& Yue, 2004). Although not diagnostic, abnormalities were observed that distinguished the ill and healthy groups.

We have conducted a co-twin control study of monozygotic twins discordant for CFS to examine the pathophysiology of this illness. Among the 21 pairs of twins examined with quantified EEG, we observed that the magnitude of the delta and theta bands at $\mathrm{Fz}, \mathrm{Cz}$ and $\mathrm{Pz}$ was significantly higher in CFS twins than their healthy co-twins (T. Budzynski, personal communication, June, 2005). The co-twin control methodology is a matched pair analysis that adjusts for many genetic and environmental factors not generally considered in case-control studies (Hrubec and Robinette, 1984). This design offers a powerful alternative to traditional approaches that compare CFS patients to healthy, depressed, or sedentary control subjects. It is particularly valuable in studies of electrophysiological parameters because the number of data points generated is large, the range of values observed in normal individuals is wide, and many features appear to be heritable (Stassen, Lykken, \& Bomben, 1988; Christian, Morzarati, Norton, Williams, O'Conner \& Li, 1996). 
In this analysis, we used data from 17 of the 21 pairs to extend our findings using lowresolution electromagnetic tomography (LORETA) (Pascual-Marqui, 1999; Pascual-Marqui, Michel, \& Lehmann 1994). LORETA is an inverse solution technique that estimates the distribution of electrical neuronal activity in 3-dimensional space. It has been extensively used in electrophysiological research (Pascual Marqui, Esslen, Kochi, \& Lehmann, 2002) and evaluated independently in several laboratories (Fuchs, Wagner, Kohler, \& Wischmann, 1999; Worrell et al. 2000; Winterer et al.,2001). Our objective was to investigate if specific frequency band-pass regions and spatial locations are associated with CFS.

\section{METHODS}

Participant Recruitment. A total of 600 twins were mailed an intake questionnaire; 426 (71\%) were returned, and complete data were available for both members of 193 twin pairs (386 individuals). Twins were recruited through patient support group newsletters (58\%), CFS electronic bulletin board notices (15\%), practitioners/researchers familiar with CFS (11\%), twin organization and researchers (6\%), relatives and friends (3\%), and other sources (8\%). Each twin completed a mailed questionnaire that collected extensive data on demographics, zygosity, life style and habits, psychiatric and physical health conditions, the nature, extent, and consequences of fatigue, and a checklist of the symptoms of CFS (Fukuda et al., 1994). For the non-fatigued twin, a control version of questions was used that did not reference fatigue. A more comprehensive description of the CFS twin registry can be found elsewhere (Buchwald et al., 1999).

Psychiatric Disorders. To determine psychiatric diagnoses, the Diagnostic Interview Schedule, Version III-A (DIS) (Robins \& Helzer, 1985) was administered via telephone interview to Registry participants. The Diagnostic Interview Schedule assigns current and lifetime diagnoses by computer algorithm based on the criteria of the Diagnostic and Statistical Manual of Mental Disorders, Version III-Revised (American Psychiatric Association, 1987). A trained research assistant administered modules on major depression, dysthymia, generalized anxiety, 
panic, agoraphobia, post-traumatic stress disorder, mania, bipolar affective disorders, schizophrenia, eating disorders, somatization, and substance abuse/dependence.

Subjects. From the twin registry, 22 sets of monozygotic twins discordant for CFS were chosen for a 7-day in-person evaluation based on registry information and additional telephone screenings. Twins were required to 1 ) be at least 18 years of age; 2) be reared together; 3) be discordant for CFS (1 twin met the Centers for Disease Control and Prevention CFS criteria, the other was healthy); 4) discontinue alcohol, caffeine, and all medications known to affect sleep or cognition at least 2 weeks prior to evaluation; and 5) travel to Seattle together. One set of twins did not complete the EEG evaluation and the data from 3 others could not be used in this analysis.

Twins were classified as meeting CFS criteria (Fukuda et al., 1994) according to their responses to a CFS symptom checklist and the diagnoses generated by the Diagnostic Interview Schedule. The same inclusion and exclusion criteria (e.g., body-mass index, specific psychiatric disorders) and review processes were applied to the fatigued and healthy twins. Medical records for the last 5 years were reviewed by a physician knowledgeable about CFS for exclusionary medical conditions (including recurrent head trauma with prolonged loss of consciousness). A psychologist and an infectious disease specialist also independently reviewed the medical charts to verify illness/health status and approve twins for participation. Prior to the scheduled visit, we confirmed that the ill twin still met CFS criteria and the control twin was healthy and not fatigued.

Zygosity was initially determined using validated self-report methods (Torgersen, 1979; Eisen, Neuman, Goldberg, Rice, \& True, 1989), then confirmed by restriction fragment length polymorphisms. DNA was extracted from peripheral blood mononuclear cells, digested with Hae III, separated by agarose electrophoresis, blotted onto a nylon membrane, and hybridized with 6 variable number repeat tandem probes to determine monozygosity with a certainty of $\geq 99.9 \%$ (Keith \& Machin, 1997). 
EEG Data Collection. EEGs on each twin pair were obtained back-to back in a fully lighted room with each twin sitting upright in a small chair. The researchers were blinded to which twin had CFS. The actual testing took approximately 3 minutes for each of 3 periods, with instructions between each period, minimizing the possibility for napping or drowsiness to occur. The EEG was sampled with 19 electrodes in the standard 10-20 International placement referenced to linked ears. Data were collected for 100 2-second epochs in each of 3 conditions: eyes open, eyes closed, and eyes closed while performing a mental mathematics task (serial 7s). Eyes closed task data were then transported into the Eureka! software (Congedo, 2002), plotted, and carefully inspected for manual artifact-rejection. All episodic artifacts including eye blinks, eye movements, teeth clenching, body movements, or EKG artifact were removed from the stream of the EEG. Seventeen of the twin pairs had acceptable quality data after performing the artifacting procedure. Average cross-spectral matrices were computed for bands delta (23.5 Hz), theta $(4-7.5 \mathrm{~Hz})$, alpha1 $(8-10 \mathrm{~Hz})$, alpha2 (10-12), beta1 $(12-16 \mathrm{~Hz})$, beta2 $(16-20 \mathrm{~Hz})$, beta3 (20-24 Hz), beta4 (24-28 Hz), and beta5 (28-32 Hz).

LORETA Variables. For each twin, cross-spectral matrices were computed and averaged over 4-second epochs resulting in one cross-spectral matrix for each twin and for each of the discrete frequencies within each band. Based on previous LORETA analyses (e.g. Lubar, Congedo \& Askew, 2003), we used a rectangular window. Sliding overlapping windows (overlap 93.8\%) allowed reliable and smooth spectral estimates. The LORETA-Key software package (Pascual-Marqui, Michel, \& Lehmann, 1994) was used to compute LORETA current density in the frequency domain directly from the average cross-spectral matrix (Frei et al., 2001). This LORETA implementation incorporates a 3-shell spherical head model registered to recognized anatomical brain atlas (Talairach \& Tournoux, 1988), and makes use of EEG electrode coordinates derived from cross-registration between spherical and realistic head geometry (Towle et al., 1993). The solution space is restricted to cortical gray matter using the digitized probability atlas of the Brain Imaging Center at the Montreal Neurological Institute (Collins, Neelin, Peters, \& Evans, 1994), divided in 2394 voxels measuring $7 \times 7 \times 7$ mm. 
Data Analysis. For every frequency band and subject, the current density modules at each voxel (current density amplitude) were smoothed with a $10.5 \mathrm{~mm}$ 3-dimensional moving average filter, normalized, and finally log-transformed. Log-transformation of power estimates is routinely performed in EEG and LORETA to approximate data gaussianity (John, Prichep \& Easton, 1987). With LORETA, some smoothing is advisable to reduce anatomical and localization errors due to inter-individual differences in head geometry and electrodes placement. In general, local maxima can be visualized in slightly different locations. Spatial normalization consists of normalizing the square root of the sum of squared current density values for each subject at all voxels to equal unity. This manipulation eliminates confounding variables such as the inter-individual variability in skull thickness and electrode impedance, without constraining the analysis on relative power measures, which is the solution usually adopted in EEG studies. Current density amplitude estimates computed and pre-processed as described provided the data for statistical analysis.

To compare the current density amplitude of the CFS and healthy twins, we used the randomization-permutation multiple comparison t-sum approach (Congedo, Finos, \& Turkheimer, 2004). Data-permutation approaches can adaptively account for the correlation structure of the variables, an embedded feature of all electrophysiological measurements (Holmes, Blair, Watson \& Ford, 1996). We performed one test for each of the 9 frequency band-pass regions (delta, theta, alpha1, alpha2, beta1, beta2, beta3, beta4, and beta5). For the whole data set $(2,394 \times 9$ variables), voxel-by-voxel paired (twin-matched) t-tests were computed. A threshold of significance (if the global null hypothesis was false) was then computed by the t-sum method. For all bands, we tested the hypothesis that the mean LORETA current density amplitude of the 2 groups differed by subtracting the values for each CFS twin from that of their healthy co-twin (Congedo et al., 2004).

\section{RESULTS}

The average age for the 17 pairs of twins was 40.6 years, $88 \%$ were female, and 
$100 \%$ were white. CFS twins differed from their co-twins in the frequency of being employed $(53 \%$ versus $88 \%, p=0.034)$, current depression $(24 \%$ versus $0 \%, p=$ $0.046)$ and in educational level (13.7 versus 14.4 years, $p=0.048)$. Among the twins with CFS, $47 \%$ reported an acute onset with a flu-like illness with an average fatigue duration of 7.4 years.

The majority of the frequency band parameters were similar in the CFS and healthy twins: alpha1 (-1.83 difference), alpha2 (-2.46 difference), beta1 (2.01 difference), beta2 (-.42 difference), beta3 (-.34 difference), beta4 (2.52 difference), and beta5 (-1.94 difference). Figure 1 illustrates that the LORETA current source density in the delta $(2-3.5 \mathrm{~Hz})$ band was higher in the CFS twins than among the healthy twins in the left uncus and parahippocampal gyrus (Brodmann areas 28, 36, 38 and 20). Figure 2 illustrates that theta also was higher in the CFS group in the cingulate gyrus (Brodmann areas 24 and 32) and right precentral gyrus of the frontal lobe (Brodmann areas 6 and 8). The maximum t-statistic, or maximum t-value across the entire volume was 3.61 and 6.08 for delta and theta respectively, which suggests that the effect for theta is much stronger than the effect for delta.

\section{DISCUSSION}

LORETA is a technique that facilitates a deeper understanding of the neurological findings present in the EEG (Pascual-Marqui et al., 2002). LORETA does not assume a limited number of dipolar point sources or a distribution on a known surface, but directly computes a current distribution throughout the full brain volume. The result is a true 3dimensional tomography with localization that is preserved with a relatively low spatial resolution. A direct comparison of results obtained from fitting one and 2 dipoles with LORETA shows that the latter provides physiologically meaningful results in many situations where dipolar solutions fail (Pascual-Marqui, 1995). LORETA also offers new hypotheses on the location of higher cognitive functions in the brain (Pascual-Marqui, 
1995), an area of great interest to CFS patients and investigators. Taken together, the advantages of LORETA over surface EEG analysis include the ability to visualize anomalies in the deeper brain structures, and to specify anomalies in frequency band and spatial location.

Our study found that the CFS twins had significantly higher levels of current source density in the delta band in the left uncus and parahippocampal gyrus, cingulate gyrus, and right precentral gyrus of the frontal lobe. These findings are consistent with those obtained with quantified surface EEG techniques both in our experience (T. Budzynski, personal communication, 2005) as well as in the literature (Billiot et. al, 1997). Previous studies have found that slowing of the deeper structures of the limbic system are associated with affect. Limbic inputs from the amygdala, entorhinal and perirhinal cortex, and subiculum, may serve as a visceromotor system to provide frontal cortical influence over autonomic and endocrine function. This system appears to be involved in guiding behavior and regulation of mood. Functional imaging studies indicate that many of these areas also show volume changes and decreased glial number and density in mood-disordered subjects (Price, 1999).

One aspect of this study that deserves emphasis is the fact that the participants were carefully chosen illness discordant twins. To our knowledge, no previous studies of LORETA involving families or twins have been published. However, previous twin studies have demonstrated that many EEG features are predominantly determined by heredity (Stassen et al., 1988; Christian et al., 1996; Martinovic et al., 1997), including peak alpha frequency (Posthuma, Neale, Boomsma, \& de Gues, 2001). Of additional relevance, in a study of older adults, $11 \%$ of "normal" control subjects exhibited an abnormal EEG (Leuchter, Daly, Rosenberg-Thompson, \& Abrams, 1993), underscoring the value of exceedingly well-matched controls such as the ones we used in this study. 
This co-twin control study has several limitations related to our sample selection and methods. Solicitation by advertisement resulted in a volunteer sample of twin pairs with the potential for ascertainment problems. However, the more desirable strategy of systematically identifying twins from a well-defined population-based twin registry is not readily accomplished in the United States. Thus, how representative the twins in this study were either of twins in general, or of persons with CFS, is not known. Because our twins were adults, primarily female, drawn from community practices, and the ill twins met strict criteria for CFS, we can not generalize our findings to other samples and more specialized settings. Nonetheless, the demographic and clinical characteristics of our sample are similar to those previously described in the CFS literature. Fourth, the order of testing of the twins was not formally randomized, so that even though technicians and investigators were blinded, some unrecognized and unaccounted for biases might have resulted. Finally, of concern given the high rates of depression in CFS, we were not able to control for major depression. Due to the constraints of the procedure implemented, no other factors entered into the statistical design.

In conclusion, objective measures of delta and theta current source density differed in twins with CFS and their healthy co-twins. Because twin studies are especially well suited to the study of illnesses for which the appropriate comparison groups are not clearly defined (Hrubec \& Robinette, 1984), they offer an alternative approach to examine possible patterns of brain activity in CFS. Future research should focus on investigating larger groups of patients and control subjects. Although these results await replication by others, they provide preliminary evidence that objective electrophysiological parameters may help identify persons with CFS. If alterations in LORETA patterns in CFS can be confirmed, this technique could find clinical applications. 
ACKNOWLEDGMENTS

The authors would like to thank the participants in the University of Washington Twin Registry for their cooperation, patience, and goodwill. 


\section{REFERENCES}

American Psychiatric Association. (1987). Diagnostic and Statistical Manual of Mental Disorders, 3rd ed. (Revised). Washington DC: American Psychiatric Association.

Billiot, K. M., Budzynski, T. H., \& Andrasik, F. (1997). EEG patterns and chronic fatigue syndrome. Journal of Neurotherapy, 2:20-30.

Buchwald, D. S., Herrell, R., Ashton, S., Belcourt, M., Schmaling, K., \& Goldberg, J. (1999). The chronic fatigue twin registry: Method of construction, composition and zygosity assignment. Twin Research, 2:203-211.

Christian J.C., Morzorati S, Norton J.A. Jr, Williams C.J., O'Connor S, Li, T.K. (1996).

Genetic analysis of the resting electroencephalographic power spectrum in human twins. Psychophysiology. 33:584-591.

Collins D.L., Neelin P., Peters T.M., Evans A.C. (1994). Automatic 3D intersubject registration of MR volumetric data in standardized Talairach space. Journal of Computer Assisted Tomography. 18:192-205.

Congedo, M. (2002). EureKa! (Version 3.0) [Computer Software]. Knoxville, TN: NovaTech EEG, Inc. Freeware available at www.NovaTechEEG.com

Congedo, M., Finos, L., \& Turkheimer, F. (2004). A Multiple Hypothesis Test Procedure based on the sum of Test-Statistics, 10th Annual Meeting of the Organization for Human Brain Mapping, June 13-17, Budapest, Hungary. (Abstract) on CD.

Eisen, S., Neuman, R., Goldberg, J., Rice, J., \& True, W. (1989). Determining zygosity in the Vietnam Era Twin Registry: An approach using questionnaires. Clinical Genettics, 35:423-432.

Frei, E., Gamma, A., Pascual-Marqui, R. D., Lehmann, D., Hell, D., \& Vollenwider, F. X. (2001). Localization of MDMA-induced brain activity in healthy volunteers using low resolution brain electromagnetic tomography (LORETA). Human Brain Mapping, 14:152-165. 
Fuchs, M., Wagner, M., Köhler, T., \& Wischmann, H. A (1999). Linear and Nonlinear Current Density Reconstructions. Journal of Clinical Neurophysiology, 16:267-295.

Fukuda, K., Straus, S. E., Hickie, I., Sharpe, M. C., Dobbins, J. G., Komaroff, A. \& the International Chronic Fatigue Syndrome Study Group. (1994). The chronic fatigue syndrome: A comprehensive approach to its definition and study. Annals of Internal Medicine, 121:953-959.

Holmes A.P., Blair R.C., Watson J.D., Ford I. (1996). Nonparametric analysis of statistic images from functional mapping experiments. J Cereb Blood Flow Metab. 16:7-22.

John, E. R., Prichep, L. S, \& Easton, P. (1987). Normative data banks and neurometrics: Basic concepts, method and results of norm constructions in method of analysis of brain electrical and magnetic signals. In: Gevins, A. S. \& Remond A., eds., EEG Handbook (revised series. Vol.1), Elsevier Science Publishers: New York.

Katon W.J., Buchwald D., Simon G., Russo J.E., Mease P.J. (1991). Psychiatric illness in patients with chronic fatigue and those with rheumatoid arthritis. Journal of General Internal Medicine, 6:277-285.

Keith, L., \& Machin, G. Zygosity testing: Current status and evolving issues. (1997). Journal of Reproductive Medicine, 42:699-707.

Komaroff, A. L., \& Buchwald, D. (1991). Symptoms and signs of chronic fatigue syndrome. Reviews of Infectious Diseases, 13:S8-11.

Leuchter, A. F., Daly, K. A., Rosenberg-Thompson, S., \& Abrams, M. (1993).Prevalence and significance of electroencephalographic abnormalities in patients with suspected organic mental syndromes. Journal of the American Geriatric Society, $41: 605-611$.

Lubar, J. F., Congedo, M., \& Askew, J. H. (2003). Low-resolution electromagnetic tomography (LORETA) of cerebral activity in chronic depressive disorder. International Journal of Psychophysiology, 49:175-185. 
Manu P., Matthews, D. A., \& Lane, T. J. (1998). Depression among patients with a chief complaint of chronic fatigue. Journal of Affective Disorders, 17:165-172.

Martinovic, Z.J., Jovanovic V, Ristanovic D. (1997). Computerized EEG topography of normal preadolescent twins--correlating similarity of background activity with genetic relatedness. Brain Topography. 9:303-311.

Pascual-Marqui, R. D., Michel, C. M., \& Lehmann, D. (1994). Low resolution electromagnetic tomography: A new method for localizing electrical activity in the brain. International Journal of Psychophysiology, 18:49-65.

Pascual-Marqui, R. D. (1995). Reply to comments by Hämäläinen, Ilmoniemi \& Nunez. In Source localization: Continuing discussion of the inverse problem (W. Skrandies Ed.). ISBET Newsletter, 6:16-28.

Pascual-Marqui, R. D. (1999). Review of methods for solving the EEG inverse problem. International Journal of Bioelectromagnetism, 1:75-86.

Pascual-Marqui, R.D., Esslen, M., Kochi, K., Lehmann, D. (2002). Functional imaging with low resolution brain electromagnetic tomography (LORETA): a review. Methods \& Findings in Experimental \& Clinical Pharmacology. 24C:91-95.

Petersson, K. M., Nichols, T. E., Poline, J. B., \& Holmes, A. P. (1999). Statistical limitations in functional neuroimaging II. Signal detection and statistical inference. Philosophical Transactions of the Royal Society of London Series B-Biological Sciences, 354:1261-1281.

Price, J. L. (1999). Prefrontal cortical networks related to visceral function and mood. Annals of the New York Academy of Sciences. 877, 383-396.

Posthuma, D., Neale, M. C., Boomsma, D. I., \& de Geus, E. J. (2001). Are smarter brains running faster? Heritability of alpha peak frequency, IQ, and their interrelation. Behavioral Genetics, 31:567-579.

Robins, L. N., Helzer, J. E.. (1985). Diagnostic Interview Schedule (DIS): version III-A. St. Louis: Department of Psychiatry, Washington University School of Medicine. 
Siemionow V., Fang Y., Calabrese L., Sahgal V., Yue G.H.. (2004). Altered central nervous system signal during motor performance in chronic fatigue syndrome. Clinical Neurophysiology. 115:2372-2381.

Stassen, H. H., Lykken, D.T., Bomben, G. (1988). The within-pair EEG similarity of twins reared apart. European Archives of Psychiatry and Neurological Sciences, 237:244-252.

Talairach J, \& Tournoux P (1988). Co-Planar Stereotaxic Atlas of the Human Brain: Three-Dimensional Proportional System. Georg Thieme: Stuttgart.

Torgersen, S. (1979). The determination of twin zygosity by means of a mailed questionnaire. Acta Genet Med Gemellol (Roma), 28:225-236. XXX full journal title

Towle, V. L., Bolaños, J., Suarez, D., Tan, K., Grzeszczuk, R., Levin, D. N., Cakmur, R., Frank, S. A., \& Spire, J. (1993). The spatial location of EEG electrodes: Locating the best fitting sphere relative to cortical anatomy. Electroencephalography and Clinical Neuroscience, 86:1-6.

Winterer G., Mulert C., Mientus S., Gallinat J., Schlattmann P., Dorn H,. Herrmann W.M. (2001). P300 and LORETA: Comparison of normal subjects and schizophrenic patients. Brain Topography 13:299-313.

Wood, G. C., Bentall, R. P., Gopfert, M., \& Edwards, R. H. T. (1991). A comparative assessment of patients with chronic fatigue syndrome and muscle disease. Psychological Medicine, 21:618-628.

Worrell G.A., Lagerlund T.D., Sharbrough F.W., Brinkmann B.H., Busacker N.E., Cicora K.M., \& O'Brien T.J. (2000). Localization of the epileptic focus by low-resolution electromagnetic tomography in patients with a lesion demonstrated by MRI. Brain Topography 12: 273-282. 
Figure 1. Current Source Density Differences Delta

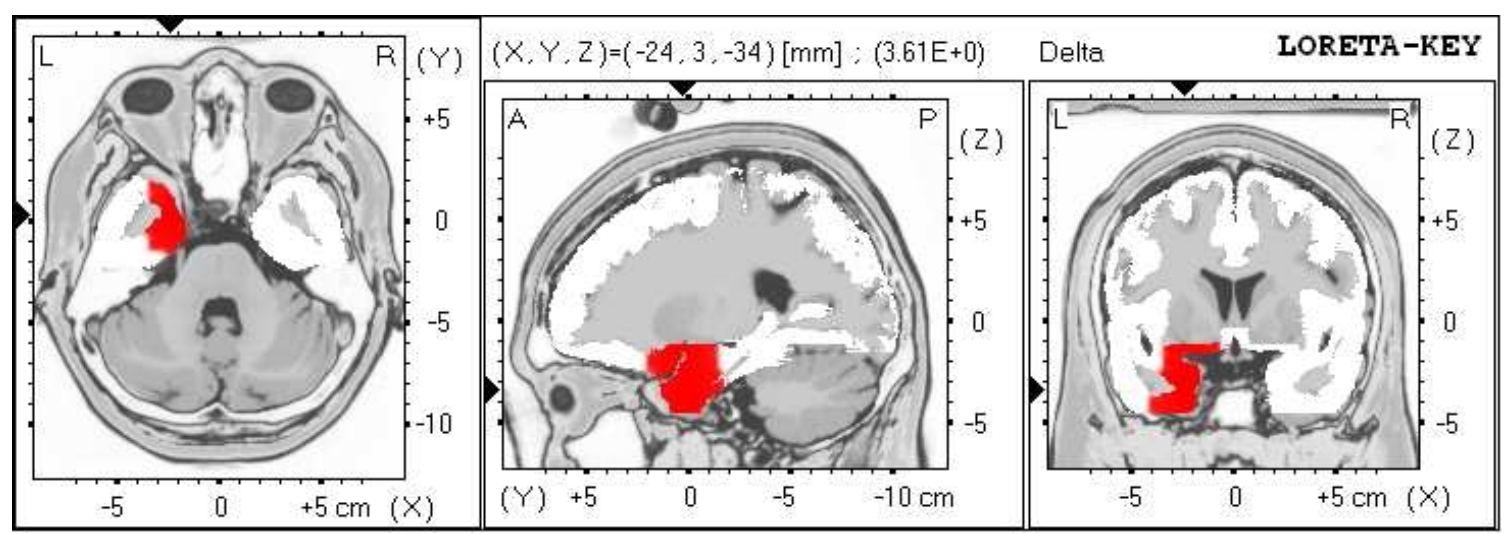

Figure 1. Significant results for Current density amplitude Analysis in the Delta band. LORETA current source density in the delta $(2-3.5 \mathrm{~Hz})$ band was higher in the CFS twins than among the healthy twins in the left uncus and parahippocampal gyrus (Brodmann areas 28, 36, 38 and 20). Coordinates and $t$-values for this voxel are printed above the picture of the sagittal section. All $t$-statistics are positive, are displayed in red (the mean of CFS group is greater than the mean of the control group). Displayed are the horizontal (left), sagittal (middle), and coronal (right) sections through the voxel with maximal $t$ statistic. This image shows significant results only. 
Figure 2. Current Source Density Differences Theta

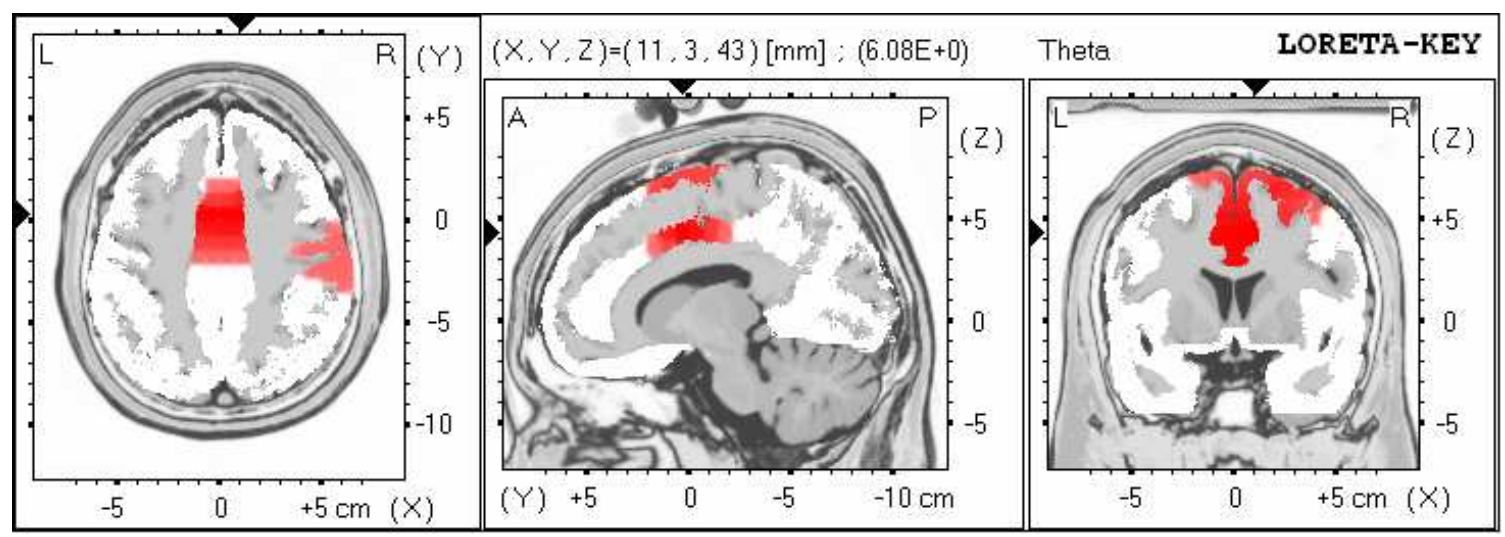

Figure 2. Significant results for Current density amplitude Analysis in the Theta band. LORETA current source density in the theta $(4-7.5 \mathrm{~Hz})$ band was higher in the CFS group in the cingulate gyrus (Brodmann areas 24 and 32) and right precentral gyrus of the frontal lobe (Brodmann areas 6 and 8 ). Coordinates and $t$-values for this voxel are printed above the picture of the sagittal section. All $t$-statistics are positive, are displayed in red (the mean of CFS group is greater than the mean of the control group). Displayed are the horizontal (left), sagittal (middle), and coronal (right) sections through the voxel with maximal $t$-statistic. This image shows significant results only. 
